\title{
Effective health services planning and management: a qualitative case study exploring health services users perspectives
}

Gigil Marme ( $\nabla$ gigil.marme@alumni.griffithuni.edu.au )

Divine Word University https://orcid.org/0000-0002-4836-5610

\section{Research Article}

Keywords: planning, management, effective health services, patients perspective,

Posted Date: August 28th, 2020

DOI: https://doi.org/10.21203/rs.3.rs-67378/v1

License: (c) (1) This work is licensed under a Creative Commons Attribution 4.0 International License.

Read Full License 


\section{Abstract}

Background: Planning and management of effective primary health services is a major problem for both the developing and developed countries. The aim of this case study is to explore health services users' perspective of effective health services delivery and understand the current challenges affecting the management of health services at the health facility.

Methods: Qualitative data were collected using semi-structured interviews with key informants representing academics, undergraduate students, administration staff and healthcare workers. The interviews covered three main areas: users' views of effective health services, current challenges affecting effective planning and management of primary healthcare services, and interventions to improve health services planning and management.

Results: Many factors exist to influence the effective delivery of health services. Patients experienced several factors that affected their abilities to access to health services. The results show that health systems and personal factors have a major influence on the management and health services delivery.

Conclusion: Poor management of health systems context has a major influence on health services delivery. This has led to poor population health outcomes especially in developing countries. Improved management and planning of health systems including infrastructures, medical supplies, healthcare workforce, medical equipment and leadership and governance and collaboration with key stakeholders' may result in improved health services management practices. If the health systems are adequately supported, the local community may experience significant improvement in health status.

\section{Background}

Effective planning and management of health services to meet the needs and expectations of patients remains a primary focus of healthcare institutions globally. Renedo \& Marston (1) argue that health services directed towards patient needs and preferences are an important aspect of quality health care and coined the terms "patient-centeredness" health services (2). Additionally, Berman, Pallas, Smith, Curry, \& Bradley (3) identified accessibility and usage of health services as an important aspect to effective health services and not necessarily availability of adequate funding and technology. Berman argue that the health systems should provide opportunities for those in need to access health services. As such, researchers also argue that patients' voices are increasingly becoming important in healthcare planning and management $(3,4)$. However, health services users' voices are not always heard and included in the planning and management of health services as part of the quality improvement activity $(5,6)$. Hence, there is a lack of data on users' perspectives of effective health services delivery in Madang Province. This study examines users and providers' perceptions of effective health services delivery and challenges affecting planning and delivery of health services at primary healthcare (PHC) facility. The study focuses on how users constructs ideas of effective health services when accessing health services at a health facility, and how typical barriers or challenges affected the effective provision of health services. 
Current research on the effectiveness of health services delivery provided insights on barriers and facilitators to provision of effective health services and how to improve or overcome the challenges $(3,7)$. However, few studies have been conducted on health services users' perceptions of effective health services in PNG site despite studies being done on practitioners and users perceptions in other developing countries such as Nepal (6). The purpose of this research is to examine users' views of effective health services and investigate barriers or challenges affecting the effective management of health services. The findings from this study may provide useful insights for health services planning and management to improve the effectiveness of health services.

\section{Methods}

\section{Study setting}

This health facility is a level one PHC facility established by a tertiary institution located in Madang Province. It served a total population of approximately 3000 within an urban setting. The health facility provides general health services including adult and children outpatient services, nurses' consultation, specialist doctors' consultation, and health risk assessments. Nurses refer major health conditions that need intensive care to the nearby Provincial Hospital. The users have the opportunity to use other private and state owned health services located within the town. Despite the small size of the health facility, their statistics reveal that many users prefer using the facility. The major causes of morbidity seeking outpatient treatment include simple cough, skin diseases and malaria. This health facility was selected because no such study was conducted to assess the effectiveness of its health services and the barriers affecting the health services from the perspective of users. The results may be useful for policy makers and university administrators for planning to make the health service delivery more effective and accessible to its users.

\section{Participants}

Twenty one adults health services users were recruited using maximum variation sampling techniques (8). The study participants are key users from the university community that frequently access the health facility (more than five times). The participants were purposefully selected based on the health facility attendance registration (8).Twenty one key informants from the university community, comprising academics, administration staff, student services and students were all given the same interview questions to contextualise the users responses. Questions were both open- ended and closed ended, to get a naturalistic and objective views of the users based on their personal situations (9). The questions directed the study participants to the main objectives of the study, to improve the effectiveness of health services delivery. Hence, the study is focused on users' responses related to their' personal experiences and decisions. The researcher emailed the study participants and sought their approval to participate in the survey. After establishing the basic contact with the study participants, survey forms were emailed to them and made appointment for an interview. To increase the sample size, two follow-ups emails were sent to participants, along with ongoing communication with staff from the health facility. 


\section{Design and procedure}

This qualitative case study used individual face-to-face semi-structured interviews with health services users (8-10). To extract in-depth insights of the phenomena under study, a guided set of open-ended questions were developed according to literature review and best evidence available to (i) understand users' perspective of how they construct concepts of effective health services, (ii) to identify personal and health systems factors affecting the management and delivery of health services at the health facility. Health services users with access to health services for more than five times were recruited to participate in the study since they are most likely to understand issues affecting the management of health services provided by the health clinic. Data were collected between April and July 2019.

\section{Data collection}

Qualitative data were collected through semi-structured interviews with the health services users and explored their accessibility, uses and experiences of healthcare provision and current issues affecting the delivery of health services (11). The key informants were asked about their experiences of accessing the health services and the difficulties experienced while accessing these services with a focus to identify factors to improve the delivery of health services and make it more effective. The interviews with the participants continued until issues reported were identified to have theoretically saturated and no new relevant information seem to emerge (7)

\section{Data analysis}

An inductive approach to data analysis was done starting with predetermined questions and collected empirical data which are used to suggest a model of care to improve the delivery of health services at the clinic $(9,11)$. All semi-structured interviews were transcribed verbatim and coded using QDA Miner Lite software (12-14). The qualitative data were transcribed, translated, and analysed by the researcher using thematic framework (8). Data were examined and record patterns (themes) within the data through the process of coding to establish meaningful patterns. I applied the six stages as outlined by Braun and Clarke: (i) becoming familiar with the data, (ii)produces initial codes,(iii) look for themes within the codes, (iv)review the codes, (v)refine the themes and (vi) produce the final report $(8,15,16)$

\section{Ethical consideration}

The study received ethical approval from the Divine Word University, Faculty of Medicine and Health Science Research Committee (approval number: FRC/MHS/01-19) and Vice President Student Affairs, Divine Word University to access the health facility and to collect data at the health facility site. Informed consent were also obtained from the study participants prior to their involvement in the study.

\section{Study limitations}

This study has several limitations. First, as is common with case studies, the study site is limited to a small health facility (level 1 health facility). Therefore, the findings may not be automatically generalised 
to other healthcare settings. However, delivering effective health services is a global issue affecting all healthcare institutions. Therefore, the results may be useful for health managers, policy makers and healthcare workers to improve the delivery and management of health services in PNG. Second, the health facility is a private clinic and as is common with private health care institutions, there is a high cost to access health services. Thus, the participants are university staff and students with high level of socioeconomic status and therefore may not reflect the views of people with lower socioeconomic status

in the community. The researcher was unable to recruit patients from the nearby community that uses the health facility due to time limitations. Therefore, there may have been a selection bias in the sample. However, the purpose of the study was to identify areas that need improvement to health services so the key informants from the university may provide useful information that may benefit the nearby community.

\section{Purpose of the study}

The purpose of this qualitative exploratory study was to explore patients and healthcare practitioners' experiences of the effectiveness of health services management and identify current challenges affecting the management of health services. The study used a qualitative study design to contribute to the knowledge of the effectiveness of health services management and its problems. The study provides new model of healthcare to manage health services effectively to improve population health outcomes rather than reactive approach.

\section{Results}

In total, 21 one-on-one semi-structured interviews with different health services users were performed between April and July 2019. A total of 21 key informants (10 academics, 7 students, 2 administration staff, and 2 student services staff took part in the study. Majority $(14 / 21,67 \%)$ of the participants were female compared with 7 male participants with an average age ranging from 20 - 79 years old. Several key themes related to effective health services planning and management emerged from this study.

\section{Theme 1: General perceptions towards effective health services}

Participants viewed accessibility to health basic health services as an important concept of effective health services delivery. The semi-structured interviews with key informants indicated that the lack of accessibility to health services is a barrier to good health outcome. They argue that the absence of health services such as basic laboratory facilities at the clinic is stressful and affected their health seeking behaviour.

"I suggest that the pathology section to check for Malaria parasites be available here at the clinic instead having patients walk all the way to Paramed for check-up then back to the clinic to get medication" (Participant 001). 
The availability of health services for those in need is an indication of effective health services.

Participants point out that health services are effective when essential health services are available at the health facility and are able to access their services when they are sick. All $(100 \%, 21 / 21)$ the participants complained that availability of health services after hours, from $5 \mathrm{pm}$ to $7 \mathrm{am}$, weekends and public holiday is actually difficult. About $81 \%(17 / 21)$ of the participants suggested that it is necessary to consider putting some form of information about who to consult during these times. About three $(3,14 \%)$ participants reported that even students find it easy to use the services. Despite these setbacks, they all concur that the health services provided are of high quality.

"A doctor must be available on site 5 days a week and must be available after hours and during weekends for emergency services. "Not all of our families and us have vehicles to move. Going to Pharamed campus for tests is not as easy as what many think. Total inconvenience" (Participant 004), "There is not even any form of notice who to contact during emergencies" (Participant 004, 011).

\section{Theme 2: Health Workers conduct and attitude towards patients}

Findings from the semi-structured interviews reveal that health care workers' (HCWs) professionalism and attitude was influential on their health seeking behaviour. Nineteen $(19,99 \%)$ health services users highly commended the health workers for their positive attitudes, and actions while seeking treatment at the clinic. They maintained that the health workers are easily approachable and relate well to patients when they are at the clinic. Majority (99\%) of the interviewees showed much more positive attitudes towards HCWs. About $10 \%(2 / 21)$ of the interviewees perceived that HCWs positive attitudes' and friendly towards patients has developed trust and motivated them to seek medical help at the clinic. They maintained that the trust between the health staff and patients play an important role to accessing health services. The interviewees emphasise that because of the staff positive attitude, they are open to provide their full medical problems without fear of stigma and discrimination.

"Services provided is superb! Staff are very helpful" (Participant 001). "I trust the nurses so I want to tell them about all my health problems, because I know they will help me" Participant 0020). "The clinic is convenient for me to access and also it has excellent team of clinical staff. The clinic has a friendly receptionist who is also very helpful. Most of the times, I book in to see medical doctors who are polite, professional and provide the best care and advice. On few times, I had appointment with the nursing staff. In addition, nursing staff are knowledgeable and provide best care. I have no negative comments but praises" (Participant 006).

\section{Theme 3: Health workers' skills and expertise}

The participants in this study viewed health workers' skills and expertise as animportant element of effective health services. The health workers' expertise and skills are key to proper diagnosis of medical conditions and prescribing appropriate treatment. About 19\% (4/21) of the participants reported that the nurses are very knowledgeable and make accurate diagnosis and prescribed right treatment. 
Participants' experiences reveal that healthcare practitioners' knowledge, experiences and skills are also an important aspect of good health services management. The participants highly commended the health workers for their expertise in the clinical management of health problems. Adequate training and education of health workers' problem a key role and may lead to improved health outcomes of those in need. The results from the participants' experiences affirm that right diagnosis and treatment are results for adequate training and education of health professionals. Three participants said this about HCWs knowledge and skills:

"On few times, I had appointment with the nursing staff. Also, nursing staff are knowledgeable and provide best care. I have no negative comments but praises" (Participant 006). "... she is very knowledgeable and well inform of and her diagnosis is accurate" (Participant 007). "...treatment given also is effective, meaning you get healed once the staff start you on treatment for whatever condition you present to them" (Participant 008).

In contrast, they argue that the absence of specialist nurses and doctors is a barrier to effective health services. They contended that they needed technical information to manage certain health problems. They cited the absence of a pediatrician as limitation to quality health services. One participant said this about the unavailability of specialist medical officers: "We have specialist doctors on campus except a Pediatrician - we have so many children especially babies on campus and if we had a Pediatrician, it would be very good for us. Babies need special care and special attention and it is vital we have a specialist for children on campus. When my baby gets sick, I do not work and this affects my students, especially those of us who are teaching. With advice from a pediatrician we will feel that our babies are okay and that we can go to work otherwise, we stake sick leave and nurse them at home or go somewhere else to look for help" (participant 002).

\section{Theme 4: Level of care}

The quality of healthcare is an important indicator of effective health services. Different users can define quality in the healthcare systems. However, from the users' perspective in this study, quality healthcare means best, timely, fast and good staff behaviour. About $81 \%$ (17/21) of the participants reported that the general level of care given at the clinic are outstanding compared to other public health facilities.

Furthermore, 76\% (15/21) participants reported that they have no major problems accessing health services and consulting health workers to seek medical treatment. Additionally, they feel comfortable talking to staff, as they are responsive to their needs. The participants stated that the quality of health services they receive is of a high standard. They said that they are given medical treatment on time and do not wait longer at the clinic. The healthcare workers are caring as this is reflected in the level of care given to patients and high rate of recovery after taking the prescribed treatment. One participant said that:

"Services provided at clinic are the best by any standard as far as PNG's public health care system is concerned. Best in the sense that service are provided on time, patients do not wait on long queues, staff are friendly and polite and they talk to you nicely. Treatment given also is effective, meaning you get 
healed once the staff start you on treatment for whatever condition you present to them" (Participant, 008).

\section{Theme 5: Health systems challenges affecting the delivery of health services}

The information below outlines challenges affecting the delivery of health services based on the survey and interview data collected from the key informants.

\section{Sub-Theme 5.1 Lack of continuity of health services}

The survey of semi-structured interview results show that majority of the participants perceived access to health services from $5 \mathrm{pm}$ to $7 \mathrm{am}$, weekends and public holidays is problematic. As shown in graph 2 , about $67 \%(14 / 21)$ of the interviewees strongly agree while $19 \%(4 / 21)$ also agree that access to health services is difficult during these times. Others, about $56 \%(12 / 21)$ even reported that there is no contact information about who to consult during emergencies. HCWs affirmed that there is no formal arrangement with the university management/administration for them to work after the normal working hours, for instance, $5 \mathrm{pm}-7 \mathrm{am}$, weekends and public holidays. As such, they affirm that they do not work shift and overtime and no contact information is posted for patients to access health workers. They explain that their employment contract allow them to work from 8am to $5 \mathrm{pm}$ daily during weekdays and not on weekends and public holidays. They iterated that when either sick patients staff from the university or students approached them, they make a decision to attend or not to attend. Another health worker maintains that developing good public relations with a health worker may make is possible to contact him/her on weekends. Additionally, another HCW noted that employment of a medical officer at the clinic to provide the strategic directions of the health services might address the lack of accessibility of health services.

"Only if you happen to know one of the staff it is easy to call upon them in the weekend" (HCW 001), "It need a part-time medical officer and leader who will plan strategic development of services" (HCWs 002).

"I sign the contract to work from 8 o'clock am to 5 o'clock pm from Monday to Friday. I am not allowed to work after hours, weekend and on public holidays. But I do help patients when they come to my house as part of my community services since I started work here" (HCW 001)

"Sadly, we do not have an emergency number that we can call in emergency. And we also do not know if a clinic staff/ or doctor available for assistance in emergency after hours and on weekends" (Participant 008).

\section{Sub-Theme 5.2: Inadequate health services infrastructures'}

Inadequate health services infrastructure is another important factor affecting the delivery of health services. The survey found that the lack of laboratory services at the clinic has affected the effectiveness 
of health services management. About $52 \%$ (14/21) strongly agree that the lack of access to laboratory services is a major barrier to effective health service. In addition, employment and availability of specialist medical doctors also contribute to effective health services delivery. About $45 \%(9 / 21)$ of the participants perceived inadequate specialist doctors as a barrier to quality health services. A further $35 \%$ $(7 / 21)$ of the participants reported that lack of appropriate health services is an obstacle. Interviews with the key informants indicated that patients are frustrated due to lack of laboratory services. The participants noted that it is time-consuming travelling to the pathology for simple blood examinations. The perceptions of key informants were based on their personal experiences of trying to access health services at the clinic and expressed disappointed over the absence of basic health services. My interview with healthcare providers agree with the patients and suggested that the clinic should start with some basic blood examination.

Furthermore, one participant perceived that there is an increased number of referral to the pathology due to the absences of laboratory services.

"In terms of laboratory services, start with malaria microscopy. As this is the most common lab investigation ordered by officers at the clinic and clients have to go all the way to Paramed to do the test and bring the results back down to the clinic. It is a very time consuming exercise" (HCW 005).

"I suggest that the pathology section to check for Malaria parasites be available here at the clinic instead having patients walk all the way to Paramed for check-up then back to the clinic to get medication" (Participant 001).

\section{Discussion}

The study has explored users and providers' perceptions of effective health services and obtained multiple theories of effective delivery of health service. The goal of the healthcare system is to provide quality, affordable, and accessible health services to its citizens to improve their health status $(6,17)$. Berman et al. (3) explained that effective health services delivery and performance means that there is an opportunity for those in need to access and use health services. Furthermore, Berman emphasised that quality health services require adequate inputs such as efficient allocation of limited resources, and adapt to new changes and technologies. There are several interpretations of the same event, but the most popular is made by Berman et al. (3) and Suman \& Bhutani (18) that by utilising a combination of financial, physical, and human resources may promote organisational effectiveness. Considering all the options, Berman concluded that improving organisational performance is a significant assurance to effective delivery of healthcare. The data in this study suggests that health services users view effective health care differently, however, common themes emerging from the participants includes availability, accessibility, affordability, and acceptability (6).

This study demonstrates a correlation between similar study done by Global Health Workforce Alliance (19). The analysis confirms that health systems factors have a major influence on the effective delivery of health services (7). Furthermore, several implications are related to participants' narratives such as 
travelling time, missing work, and risk walking to nearby health facilities due to non-availability of healthcare at the local clinic. This data suggests that rather than improving health, the absence of basic healthcare erodes individuals' efforts to seek medical care. The study demonstrates a correlation between health systems barriers and availability, accessibility, and affordability of health services. Therefore, research has shown that efficient organisational policies to manage human resource and health technology may promote organisational effectiveness.

Furthermore, the capacity of the organisation to deliver institutional programs is important for organisational effectiveness (20). Thus, managing its human resources is an essential aspect of this organisational effectiveness (21). The results in this study show that the institution loses its ability to coordinate its health workers to sustain the delivery of health services at certain times due to the closure of the health facility. This result support the notion put forward by Statford (22) that employees of the organisations should be adequately managed to perform the institutional and programs activities. Statford raised this critical questions "does the organisation have the people, skills, space, funding, and other stuff necessary to carry out institutional and programmatic activities? "(p.14). Furthermore, Statford point out that organisations should collect data of its performance and use the data to manage change and be willing to alter its policies when necessary. Looking more closely, it is apparent that the institution has not been effectively implementing its human resource management policy to coordinate its health care workers to keep the health facility operational throughout the week. In this instance, the general rule of overtime management is important and may improve health services delivery leading to improved health outcomes. The arguments discussed conclusively support the point of view that overtime allowance may be considered for health workers to work after hours, weekends and on public holidays.

\section{Methodological strengths and limitations}

This study has some limitations. The participants were generally from the university, and residents from nearby community were not interviewed due to time limitation. Additionally, the users are academics and therefore, were committed within their end of semester results and therefore unable to conduct follow up face-to-face interviews. As is common with qualitative case studies, this study is limited to a small primary healthcare facility and the results cannot be automatically generalised to other settings in PNG. However, delivering effective health services is a global phenomenon and is well articulated in other studies (3), and therefore, policy makers, health managers and other health workers in other health settings in PNG may find this study useful for health services planning and management.

\section{Conclusion}

This study provides useful information into health services users perspectives towards provision of effective health services management. If health services providers, healthcare planners and clinicians consider patients' needs, expectation while planning, and delivering health care in the health systems, huge satisfaction and acceptance will be achieved. At the optimum case, this will lead to improved 
patient accessibility and positive medical health outcomes. This study argues that to provide effective health services, it is equally essential to ensure that an efficient enabling environment such as effective operational policy is available to clearly provide directions to control the operations of the health facility. It is unclear in the study how workplace policy or human resource policy influences the organisations performance. Therefore, there is a need to do further research on this research question: How does workplace policies improve staff performance in the health system in PNG? Finally, the results of this study may be useful for health managers, policy makers and clinicians at both the district, province and national level to improve the delivery and management of primary healthcare services.

\section{Declarations}

\section{Conflict of interest}

The author declares that there is no conflict of interest in this research. Australia Awards Fellowship, 2019 , funds the research project.

\section{References}

1. Renedo A, Marston C. Developing patient-centred care: An ethnographic study of patient perceptions and influence on quality improvement. BMC Health Serv Res. 2015;15(1):15-7.

2. Institute of Medicine. The rural health care delivery system. In: Quality Through Collaboration: The Future of Rural Health Care. Washington, D.C.: The National Academies; 2005. p. 1-267.

3. Berman P, Pallas S, Smith AL, Curry L, Bradley EH. Improving the Delivery of Health Services: A Guide to Choosing Strategies. Health, Nutrition and Population (HNP) Discussion Papers. 2011. 1-78 p.

4. Smith J, Scammon D, Beck S. Using patient focus groups for new patient services. J Qual Patient Saf. 1995;21(1):22-31.

5. Crawford M. User involvement in the planning and delivery of mental health services. 2001;410-4. Available from: d:/docs/_Tavi-Port/Lucy/2002/Notes from the WLMHT 6th Annual R\&D conf.doc

6. ; Regmi K. Effective health services: Perspectives and perceptions of health service users and healthcare practitioners. Prim Heal Care Open Access. 2012;02(03).

7. Wong EL, Yam CH, Cheung AW, Leung MC, Chan FW, Wong FY, et al. Barriers to effective discharge planning: A qualitative study investigating the perspectives of frontline healthcare professionals. BMC Health Serv Res. 2011;11.

8. Jane R, Jane L. Qualitative research practice A Guide for Social Science Students and Researchers. Choice Rev Online. 2003;42(04):42-2286-42-2286.

9. Methodological frameworks and sampling in qualitative research. Oxford; 2006. 5-22 p.

10. Aspers P. Empirical phenomenology: A qualitative research approach. Indo- Pacific J Phenomenol. 2009;9(2):1-12. 
11. Hall E, Chai W, Albrecht JA. A Qualitative Phenomenological Exploration of Teachers'Experience With Nutrition Education. Am J Heal Educ. 2016;47(3):136- 48.

12. Maguire, M., Delahunt B. Doing a thematic analysis: A practical, step-by-step guide for learning and teaching scholars. AISHE-J All Irel J Teach Learn High Educ [Internet]. 2017;9(3):3135-40. Available from: http://ojs.aishe.org/index.php/aishej/article/view/335\%0Ahttp://ieeexplore.ieee.org/document/6746227/

13. Abbas T, Charles T. SAGE handbook of mixed methods in social and behavioural research. 2 nd ed. California: SAGE Publication; 2010. 2010

14. Agee J. Developing qualitative research questions: A reflective process. Int J Qual Stud Educ. 2009;22(4):431-47.

15. Braun V, Clarke V. Using thematic analysis in psychology. Qualitative. Qual Res Psychol. 2006;3(2):77.

16. Young B, Hren D. Presentation slide: Introduction to qualitative research methods. Second MiRoR Train Event. 2017;

17. New Public Health. National health systems. In: The New Public Health. 3rd ed. Elsevier;

18. Suman P, Bhutani S. Availability of health services in Himachal Pradesh. Int J Adv Res Dev [Internet]. 2017;2(6):231-8. Available from: advancedjournal.com

19. Global Health Workforce Alliance. What do we mean by availability, accessibility, acceptability and quality (AAAQ) of the health workforce? [Internet]. World Health Organisation. 2019 [cited 2019 Sep 18]. p. 1. Available from: https://www.who.int/workforcealliance/media/qa/04/en/ ?

20. Boren SA usti., Moxley D. Systematically reviewing the literature: building the evidence for health care quality. Mo Med. 2015;112(1):58-62.

21. Goudge J, Gilson L, Russell S, Gumede T, Mills A. Affordability, availability and acceptability barriers to health care for the chronically ill: Longitudinal case studies from South Africa. BMC Health Serv Res. 2009;9(May 2014).

22. Statford B. Assessing Organizational Capacity: The Why and How. Assess Organ Capacit why How. 2015;

\section{Tables}

\section{Table 1 Characteristics of study participants}


Sex

Male

Female

7

$33 \%$

Missing data

14

$67 \%$

Age in years $(n=21)$

0

$0 \%$

$20-29$

Frequency (n) Percentage

$30-39$

6

$29 \%$

$40-49$

3

$14 \%$

$50-59$

6

$29 \%$

$60-69$

1

$5 \%$

$70-79$

2

$9 \%$

Missing data

1

Highest level of education

2

$5 \%$

Certificate

Frequency(n) Percentage

Diploma

0

1

Bachelor

4

Masters

7

PhD

3

Student

6

0

$0 \%$

Missing data

Frequency(n) Percentage

Administration

2

Student Services

3

Academic

10

$5 \%$

Students

6

$19 \%$

$33 \%$

Religious Affiliation

Frequency(n) Percentage

Catholic

4

$19 \%$

Lutheran

4

$19 \%$

SDA

Pentecostal

5

$24 \%$

United Church

1

$5 \%$

EBC

2

$10 \%$

Missing data

1

$5 \%$

3

$14 \%$

Number of times attended health services Frequency(n) Percentage 
Less than five times

5

$24 \%$

More than five times

16

$76 \%$ 